\section{Strahlentherapie kostet keine Lebensjahre}

Um den Kehlkopf und damit die Sprachfähigkeit zu erhalten, entscheiden sich viele Patienten mit Larynxkarzinom gegen Laryngektomie und für Bestrahlung. Bisher ist nicht klar, wie sich dies auf ihre Überlebenschancen auswirkt. US-Forscher haben dies nun untersucht - mit einem beruhigenden Ergebnis.

W er sein Larynxkarzinom bestrahlen lässt, statt sich einer totalen Laryngektomie zu unterziehen, muss nicht damit rechnen, früher zu sterben: HNOSpezialisten, Radioonkologen und Statistiker der University of Rochester hatten für ihre Untersuchung die Daten analysiert von 13.808 Patienten mit Larnyxkrebs aus dem „Surveillance, Epidemiology, and End Results (SEER) Program“ - einer Datenbank des National Cancer Institute der USA zu Krebsinzidenz und -mortalität. Bei den Karzinomen handelte es sich um nicht metastasierte glottische und supraglottische Plattenepitheltumoren, die in den Jahren 1988-2006 aufgetreten und behandelt worden waren.
Während der beobachteten Periode nahm der Anteil der Geschwülste, die nicht operiert, sondern bestrahlt wurden, kontinuierlich zu. Lokalisierte Glottiskarzinome (LGK) wurden im Zeitraum 1988 bis 1993 zu 94\%, in der Spanne von 1994 bis 1999 zu $97 \%$ und von 2000 bis $2006 \mathrm{zu}$ $98 \%$ bestrahlt. Für regional begrenzte Glottiskarzinome (RGK) lagen die Quoten bei 53,66 und $75 \%$. Bei lokalem supraglottischem Krebs (LSGK) förderte die Analyse Bestrahlungsraten von 61,83 und $94 \%$, für regionäre supraglottische Karzinome (RSGK) von 43, 55 und $78 \%$ zutage.

Ein signifikanter Unterschied in den 5-Jahres-Überlebensraten war im Vergleich der drei Zeitspannen nicht festzu- stellen. Sie lagen bei 73,76 und $78 \%$ für LGK, bei 57,51 und $56 \%$ für RGK, bei 33, 35 und $39 \%$ für LSGK sowie bei 36, 36 und $43 \%$ für RSGK. Der zunehmende Anteil an Bestrahlungen in der Therapie von Larynxkarzinomen wirkt sich demnach nicht auf die Überlebenschancen aus.

Fazit: Die Ergebnisse dieser Studie dürften Patienten und Ärzte beruhigen, die eine verkürzte Restlebenszeit als Preis für den Verzicht auf die Laryngektomie fürchten. Einen solchen Preis zu zahlen, wäre mancher indes durchaus bereit, wie eine frühere Erhebung des Massachusetts General Hospital gezeigt hat. Darin hatten Patienten geäußert, für den Erhalt der Sprache bis zu $30 \%$ ihrer Lebenserwartung eintauschen zu wollen.

Robert Bublak

Zhang $\mathrm{H}$ et al. Impact of radiotherapy on laryngeal cancer survival. Cancer. 2011 Jul 19 [Epub ahead of print].

\title{
Häufig Zweitmalignome nach Radiojodtherapie
}

Patienten mit gut ausdifferenzierten Schilddrüsenkarzinomen vom Grad T1N0 scheinen von einer adjuvanten Radiojodtherapie (RIT) nicht zu profitieren. Vielmehr steige das Risiko, an einem Zweitmalignom zu erkranken.

Z u diesem Schluss kommen Wissenschaftler um Gopalakrishna Iyer vom Memorial Sloan-Kettering Cancer Center in New York. Sie hatten Daten von insgesamt 37.176 Patienten analysiert, die an einem gut differenzierten (papillären oder follikulären) Schilddrüsenkarzinom (GDSK) erkrankt waren. 14.589 hatten eine RIT erhalten.

In der Studie wurde zweierlei beobachtet: Einmal nahmen die Verordnungen innerhalb des Beobachtungszeitraumes enorm zu: So wurden etwa von den unter 45-Jährigen im Jahr 1973 noch 3,3\% mit einer RIT behandelt; 2006 waren es schon $38,1 \%$. Ein Nutzen ließ sich indessen nicht erkennen, denn die Überlebensrate für das GDSK mit niedrigem Risiko blieb trotz vermehrter RIT konstant. Was hingegen stieg, war das Risiko, an einem Zweitmalignom zu erkranken. Die standardisierte Inzidenzrate für
Zweitmalignome betrug nach RIT 1,18 - unter den Patienten, die kein Radiojod erhalten hatten, war kein erhöhtes Risiko festzustellen. Dies entspricht einem absoluten Risikoüberschuss von 11,9/10.000 Patientenjahre. Aufgetreten waren Krebserkrankungen der Speicheldrüsen (SIR 3,84), Leukämien $(2,86)$, Nierenkrebs $(2,62)$, Melanome $(1,61)$ und Lymphome $(1,44)$.

In der Gruppe der unter 45-Jährigen lag das Risiko, einen zweiten Primärtumor zu entwickeln, nach RIT $21 \%$ höher, entsprechend 4,5 zusätzlichen Krebsfällen je 10.000 Patientenjahre.

Fazit: Für Patienten unter 45 Jahren mit intrathyreoidalen T1N0M0-Tumoren < $2 \mathrm{~cm}$ ist der Nutzen adjuvanter RIT allenfalls minimal", so Iyer und Kollegen. Zusammen mit der Gefahr, an einem zweiten Primärtumor zu erkranken,

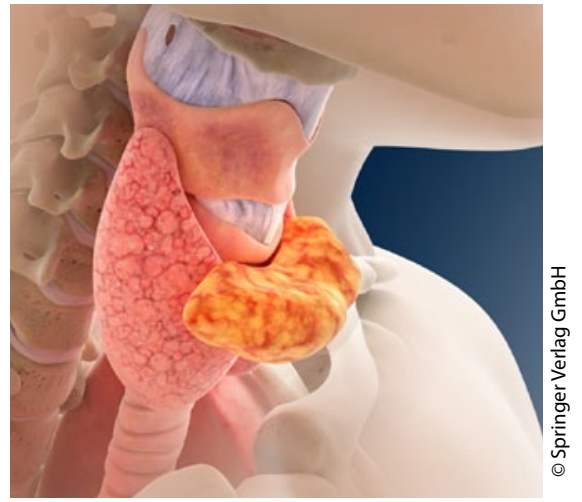

Wissenschaftler vom Sloan-Kettering Cancer Center warnen vor der Radiojodtherapie bei GDSK-Patienten mit niedrigem Risiko. In der Leitlinie der Deutschen Gesellschaft für Nuklearmedizin gilt sie noch als „Standardprozedur"

forme dies ein überzeugendes Argument dafür, die RIT in dieser Population einzuschränken.

Robert Bublak

lyer NG et al. Rising incidence of second cancers in patients with low-risk (T1N0) thyroid cancer who receive radioactive iodine therapy. Cancer. 2011; $117: 4439-46$ 